\title{
Erratum to: Analysis of 62 synthetic cannabinoids by gas chromatography-mass spectrometry with photoionization
}

\author{
Mamoru Akutsu $^{1} \cdot$ Ken-ichi Sugie $^{1} \cdot$ Koichi Saito $^{2}$
}

Published online: 28 November 2016

(C) Japanese Association of Forensic Toxicology and Springer Japan 2016

\section{Erratum to: Forensic Toxicol}

DOI 10.1007/s11419-016-0342-9

Unfortunately, in the original publication of this article, the Table 1 was published incorrectly.

The revised Table 1 is given in the following page.

The online version of the original article can be found under doi:10.1007/s11419-016-0342-9.

Mamoru Akutsu

akutsu-mamoru@mhlw.go.jp

1 Narcotics Control Department, Kanto-Shin'etsu Regional Bureau of Health and Welfare, Ministry of Health, Labour and Welfare, 1-2-1 Kudan-Minami, Chiyoda-ku, Tokyo 102-8309, Japan

2 Department of Analytical Chemistry, Faculty of Pharmaceutical Sciences, Hoshi University, 4-41 Ebara 2-chome, Shinagawa-ku, Tokyo 142-8501, Japan 
Table 1 Grouping of the 13 types of synthetic cannabinoids according to mass spectra by gas chromatography-mass spectrometry in photoionization mode

Group 1 Molecular ion only (35 compounds)

(1) Naphthoylindoles (19 compounds)

AM2232, EAM-2201, JWH-007, JWH-015, JWH-016, JWH-019, JWH-020, JWH-022, JWH-072, JWH-080, JWH-081, JWH-098, JWH-

149, JWH-182, JWH-210, JWH-213, JWH-398, JWH-412, AM1220 azepan isomer

(2) Carboxamide derivatives (5 compounds)

SDB-006, APICA, APICA $N$-(5-fluoropentyl) derivative, MN-18, 5-fluoro-MN-18

(3) Benzoylindoles (5 compounds)

AM679, AM694, RCS-4, RCS-4 ortho isomer, AM2233 azepan isomer

(9) Naphthoylindazoles (2 compounds)

THJ-018, THJ-2201

(10) Naphthoylpyrroles (2 compounds)

JWH-307, JWH-030

(12) Naphthoylbenzimidazole (1 compound)

FUBIMINA

(13) Naphthoylnaphthalene (1 compound)

CB-13

Group 2 Molecular ion with smaller fragment ion(s) (18 compounds)

(2) Carboxamide derivatives (5 compounds)

APINACA, APINACA $N$-(5-fluoropentyl) derivative, NNE1, 5-fluoro-NNE1, ADBICA

(4) Cyclopropyles (4 compounds)

A-836339, FUB-144, UR-144, XLR-12

(7) Quinolinyl carboxylates (3 compounds)

BB-22, PB-22, 5-fluoro-NPB-22

(5) Phenylacetylindoles (2 compounds)

JWH-250, JWH-251

(6) Carboxyindoles (2 compounds)

MEPIRAPIM, AB-001

(8) Naphthoyl carboxylate (1 compound)

5-Fluoro-SDB-005

(11) Cyclohexylphenol (1 compound)

CP-47,497

Group 3 Smaller molecular ion with a fragment ion as base peak (9 compounds)

(2) Carboxamide derivatives (3 compounds)

AB-CHMINACA, AB-FUBINACA, AB-PINACA

(5) Phenylacetylindoles (2 compounds)

JWH-203, cannabipiperidiethanone

(1) Naphthoylindole (1 compound)

AM1220

(3) Benzoylindole (1 compound)

AM2233

(6) Carboxyindole (1 compound)

AM1248

(8) Naphthoyl carboxylate (1 compound)

FDU-PB-22 